\title{
Multi-Parametric Families of Real and Non Singular Solutions of the Kadomtsev-Petviasvili I Equation
}

\author{
Pierre Gaillard ${ }^{1}$ \\ ${ }^{1}$ Institut de mathématiques de Bourgogne, Université de Bourgogne, 9 avenue Alain Savary, BP 47870, 21078 Dijon Cedex, France
}

\author{
Article Info \\ Keywords: Kadomtsev-Petviashvili \\ equation, rational solutions \\ 2010 AMS: 33Q55, 37K10, 47.10A-, \\ 47.35.Fg, 47.54.Bd \\ Received: 4 August 2021 \\ Accepted: 16 December 2021 \\ Available online: 30 December 2021
}

\begin{abstract}
Multi-parametric solutions to the Kadomtsev-Petviashvili equation (KPI) in terms of Fredholm determinants are constructed in function of exponentials. A representation of these solutions as a quotient of wronskians of order $2 N$ in terms of trigonometric functions is deduced. All these solutions depend on $2 N-1$ real parameters.

A third representation in terms of a quotient of two real polynomials depending on $2 N-2$ real parameters is given; the numerator is a polynomial of degree $2 N(N+1)-2$ in $x, y$ and $t$ and the denominator is a polynomial of degree $2 N(N+1)$ in $x, y$ and $t$. The maximum absolute value is equal to $2(2 N+1)^{2}-2$.

We explicitly construct the expressions for the first third orders and we study the patterns of their absolute value in the plane $(x, y)$ and their evolution according to time and parameters. It is relevant to emphasize that all these families of solutions are real and non singular.
\end{abstract}

\section{Introduction}

We consider the Kadomtsev-Petviashvili I equation (KPI)

$$
\left(4 u_{t}-6 u u_{x}+u_{x x x}\right)_{x}-3 u_{y y}=0
$$

where subscripts $x, y$ and $t$ denote partial derivatives.

This equation was introduced by Kadomtsev and Petviashvili [1] in 1970. It is considered as a model in hydrodynamic for surface and internal water waves [2] or in nonlinear optics [3]. Dryuma showed in 1974 how the KP equation could be written in Lax form [4].

Manakov, Zakharov, Bordag and Matveev first constructed rational solutions in 1977 [5] and two month later Krichever published other solutions [6].

In the frame of algebraic geometry, Krichever constructed for the first time in 1976 [7] the solutions to KPI equation in terms of Riemann theta functions and a little later, it was done by Dubrovin [8].

Others rational solutions of the KPI equation were obtained. For example, one can quote of the studies of Krichever in 1978 [9], Satsuma and Ablowitz in 1979 [10], Matveev in 1979 [11], Freeman and Nimmo in 1983 [12, 13], Matveev in 1987 [14], Pelinovsky and Stepanyants in 1993 [15], Pelinovsky in 1994 [16], Ablowitz, Villarroel, Chakravarty, Trubatch [17-19] in 1997-2000, Biondini and Kodama [20-22] in 2003-2007.

We give in the following three types of representations of the solutions to the KPI equation : first, in terms of Fredholm determinants of order $2 N$ depending on $2 N-1$ real parameters in function of exponentials, then in terms of wronskians of order $2 N$ with $2 N-1$ real parameters in function of some trigonometric functions.

In a third representation, real rational solutions of order $N$ depending on $2 N-2$ real parameters are constructed and they can be written as a ratio of two polynomials; the numerator is a polynomial in $x, y$ and $t$ of degree $2 N(N+1)-2$ and the denominator a polynomial in $x, y$ and $t$ of degree $2 N(N+1)$.

So we get rational real and non singular solutions to the KPI equation at each order $N$ depending on $2 N-2$ real parameters. We present explicit rational solutions and the representations of their absolute value in the plane of the coordinates $(x, y)$ according to the $2 N-2$ real parameters $a_{i}$ and $b_{i}(1 \leq i \leq N-1)$ and time $t$ for the first three orders. 


\section{Families of solutions of order $N$ depending on $2 N-1$ real parameters in terms of Fredholm determinants to the KPI equation}

We define the numbers $\lambda_{v}, \kappa_{v}, \delta_{v}, \gamma_{v}, x_{r, v}, e_{v}$ depending on a real number $\varepsilon$ by

$$
\begin{aligned}
& \lambda_{j}=1-2 \varepsilon^{2} j^{2}, \lambda_{N+j}=-\lambda_{j}, \quad \kappa_{j}=2 \sqrt{1-\lambda_{j}^{2}}, \quad \delta_{j}=\kappa_{j} \lambda_{j}, \quad \gamma_{j}=\sqrt{\frac{1-\lambda_{j}}{1+\lambda_{j}}}, \\
& x_{r, j}=(r-1) \ln \frac{\gamma_{j}-i}{\gamma_{j}+i}, r=1,3, \quad \tau_{j}=-12 i \lambda_{j}^{2} \sqrt{1-\lambda_{j}^{2}}-4 i\left(1-\lambda_{j}^{2}\right) \sqrt{1-\lambda_{j}^{2}}, \\
& \kappa_{N+j}=\kappa_{j}, \quad \delta_{N+j}=-\delta_{j}, \quad \gamma_{N+j}=\gamma_{j}^{-1}, \quad x_{r, N+j}=-x_{r, j},, \quad \tau_{N+j}=\tau_{j} \\
& e_{j}=2 i\left(\sum_{k=1}^{1 / 2 M-1} a_{k}(j e)^{2 k+1}-i \sum_{k=1}^{1 / 2 M-1} b_{K}(j e)^{2 k+1}\right), \\
& e_{N+j}=2 i\left(\sum_{k=1}^{1 / 2 M-1} a_{k}(j e)^{2 k+1}+i \sum_{k=1}^{1 / 2 M-1} b_{k}(j e)^{2 k+1}\right), \\
& a_{j}, b_{j} \text { real numbers, } \quad \varepsilon_{j}=1, \quad \varepsilon_{N+j}=0, \quad \varphi \text { a real number } \\
& 1 \leq j \leq N .
\end{aligned}
$$

Then we have the following statement:

Theorem 2.1. Let $v(x, y, t)$ be the expression defined by

$$
v(x, y, t)=\frac{\operatorname{det}\left(I+D_{3}(x, y, t)\right)}{\operatorname{det}\left(I+D_{1}(x, y, t)\right)},
$$

with I the unit matrix and $D_{r}=\left(d_{j k}^{(r)}\right)_{1 \leq j, k \leq 2 N}$ the matrix

$$
d_{v \mu}^{(r)}=(-1)^{\varepsilon_{v}} \prod_{\eta \neq \mu}\left(\frac{\gamma_{\eta}+\gamma_{v}}{\gamma_{\eta}-\gamma_{\mu}}\right) \exp \left(i \kappa_{v} x-2 \delta_{v} y+\tau_{v} t+x_{r, v}+e_{v}\right) .
$$

Then the function defined by

$$
u(x, y, t)=-2\left(|v(x+3 t, y, t)|^{2}-1\right)
$$

is a solution to the KPI equation (1.1) depending on $2 N-1$ real parameters $a_{k}, b_{k}, 1 \leq k \leq N-1$ and $\varepsilon$.

Proof. We have proven in [23] that the function $w$ defined by (2.5)

$$
w(x, y)=\frac{\operatorname{det}\left(I+D_{3}(x, y, 0)\right)}{\operatorname{det}\left(I+D_{1}(x, y, 0)\right)} \exp (2 i y-i \varphi)
$$

is a solution to the nonlinear Schrödinger equation (2.6)

$$
i w_{y}+w_{x x}+2|w|^{2} w=0 .
$$

It can then be similarly proven that the function $v$ defined by

$$
\tilde{v}(x, y, t)=v(x, y, t) \times \exp (2 i y-i \varphi)=\frac{\operatorname{det}\left(I+D_{3}(x, y, t)\right)}{\operatorname{det}\left(I+D_{1}(x, y, t)\right)} \times \exp (2 i y-i \varphi)
$$

is a solution to the NLS equation (2.6) by considering $t$ as a parameter. We can then deduce that the function $u$ defined by (3)

$$
u(x, t)=-2\left(|v(x+3 t, y, t)|^{2}-1\right)=-2\left(|\tilde{v}(x+3 t, y, t)|^{2}-1\right)
$$

is a solution to the KPI equation, which proves the result.

\section{Families of solutions of order $N$ depending on $2 N-1$ real parameters in terms of wronskians to the KPI equation}

We denote $W_{r}(w)$ the wronskian of the functions $\phi_{r, 1}, \ldots, \phi_{r, 2 N}$ defined by

$$
W_{r}(w)=\operatorname{det}\left[\left(\partial_{w}^{\mu-1} \phi_{r, v}\right)_{v, \mu \in[1, \ldots, 2 N]}\right]
$$

We consider the matrix $D_{r}=\left(d_{v \mu}^{(r)}\right)_{v, \mu \in[1, \ldots, 2 N]}$ defined in $(2.3)$.

We consider the real parameters $a_{k}, b_{k} 1 \leq k \leq N-1$ and $\varepsilon$, and $\kappa_{v}, \delta_{v}, x_{r, v}, \gamma_{v}, e_{v}$ defined in the previous section.

Then we have the following statement 
Theorem 3.1. Let $\Phi_{r, v}$ be the functions defined by

$$
\begin{array}{ll}
\phi_{r, v}(x, y, t, w)=\sin \left(\frac{\kappa_{v} x}{2}+i \delta_{v} y-i \frac{x_{r, v}}{2}-i \frac{\tau_{v}}{2} t+\gamma_{v} w-i \frac{e_{v}}{2}\right), & 1 \leq v \leq N, \\
\phi_{r, v}(x, y, t, w)=\cos \left(\frac{\kappa_{v} x}{2}+i \delta_{v} y-i \frac{x_{r, v}}{2}-i \frac{\tau_{v}}{2} t+\gamma_{v} w-i \frac{e_{v}}{2}\right), & N+1 \leq v \leq 2 N, \quad r=1,3,
\end{array}
$$

Let $v$ be the expression defined by

$$
v(x, y, t)=\frac{W_{3}\left(\phi_{3,1}, \ldots, \phi_{3,2 N}\right)(x, y, t, 0)}{W_{1}\left(\phi_{1,1}, \ldots, \phi_{1,2 N}\right)(x, y, t, 0)}
$$

Then the function defined by

$$
u(x, y, t)=-2\left(|v(x+3 t, y, t)|^{2}-1\right)
$$

is a solution to the KPI equation (1.1) depending on $2 N-1$ real parameters $a_{k}, b_{k}, 1 \leq k \leq N-1$ and $\varepsilon$.

Proof. We have proven in [24] that the function $v$ defined by (3.4)

$$
w(x, y)=\frac{W_{3}\left(\phi_{3,1}, \ldots, \phi_{3,2 N}\right)(x, y, 0,0)}{W_{1}\left(\phi_{1,1}, \ldots, \phi_{1,2 N}\right)(x, y, 0,0)} \exp (2 i y-i \varphi)
$$

is a solution to the nonlinear Schrödinger equation (3.5)

$$
i w_{y}+w_{x x}+2|w|^{2} w=0
$$

We can similarly prove that the function $v$ defined by

$$
\tilde{v}(x, y, t)=v(x, y, t) \times \exp (2 i y-i \varphi)
$$

is a solution of the NLS equation by considering $t$ as a parameter. We can then deduce that the function $u$ defined by

$$
u(x, t)=-2\left(|v(x+3 t, y, t)|^{2}-1\right)=-2\left(|\tilde{v}(x+3 t, y, t)|^{2}-1\right)
$$

is a solution to the KPI equation which proves the result.

\section{Real and non singular rational solutions to the KPI equation of order $N$ depending on $2 N-2$ real parameters}

We construct in this section rational solutions to the KPI equation as a quotient of two determinants. We define functions of the following arguments:

$$
\begin{aligned}
& X_{v}=\frac{\kappa_{v} x}{2}+i \delta_{v} y-i \frac{x_{3, v}}{2}-i \frac{\tau_{v}}{2} t-i \frac{e_{v}}{2} \\
& Y_{v}=\frac{\kappa_{v} x}{2}+i \delta_{v} y-i \frac{x_{1, v}}{2}-i \frac{\tau_{v}}{2} t-i \frac{e_{v}}{2}
\end{aligned}
$$

for $1 \leq v \leq 2 N$, with $\kappa_{v}, \delta_{v}, x_{r, v}$ defined in the first section.

We consider the following functions:

$$
\begin{aligned}
& \varphi_{4 j+1, k}=\gamma_{k}^{4 j-1} \sin X_{k}, \quad \varphi_{4 j+2, k}=\gamma_{k}^{4 j} \cos X_{k}, \\
& \varphi_{4 j+3, k}=-\gamma_{k}^{4 j+1} \sin X_{k}, \quad \varphi_{4 j+4, k}=-\gamma_{k}^{4 j+2} \cos X_{k}, \\
& \varphi_{4 j+1, N+k}=\gamma_{k}^{2 N-4 j-2} \cos X_{N+k}, \quad \varphi_{4 j+2, N+k}=-\gamma_{k}^{2 N-4 j-3} \sin X_{N+k}, \\
& \varphi_{4 j+3, N+k}=-\gamma_{k}^{2 N-4 j-4} \cos X_{N+k}, \quad \varphi_{4 j+4, N+k}=\gamma_{k}^{2 N-4 j-5} \sin X_{N+k}, \\
& \psi_{4 j+1, k}=\gamma_{k}^{4 j-1} \sin Y_{k}, \quad \psi_{4 j+2, k}=\gamma_{k}^{4 j} \cos Y_{k}, \\
& \psi_{4 j+3, k}=-\gamma_{k}^{4 j+1} \sin Y_{k}, \quad \psi_{4 j+4, k}=-\gamma_{k}^{4 j+2} \cos Y_{k}, \\
& \psi_{4 j+1, N+k}=\gamma_{k}^{2 N-4 j-2} \cos Y_{N+k}, \quad \psi_{4 j+2, N+k}=-\gamma_{k}^{2 N-4 j-3} \sin Y_{N+k}, \\
& \psi_{4 j+3, N+k}=-\gamma_{k}^{2 N-4 j-4} \cos Y_{N+k}, \quad \psi_{4 j+4, N+k}=\gamma_{k}^{2 N-4 j-5} \sin Y_{N+k}, \\
& 1 \leq k \leq N
\end{aligned}
$$

Then we get the following result

Theorem 4.1. Let $v$ be the expression defined by

$$
v(x, y, t)=\frac{\operatorname{det}\left(\left(n_{j k}\right)_{j, k \in[1,2 N]}\right)}{\operatorname{det}\left(\left(d_{j k}\right)_{j, k \in[1,2 N]}\right)}
$$


where

$$
\begin{aligned}
& n_{j 1}=\varphi_{j, 1}(x, y, t, 0), \quad n_{j k}=\frac{\partial^{2 k-2} \varphi_{j, 1}}{\partial \varepsilon^{2 k-2}(x, y, t, 0),} \\
& n_{j N+1}=\varphi_{j, N+1}(x, y, t, 0), \quad n_{j N+k}=\frac{\partial^{2 k-2} \varphi_{j, N+1}}{\partial \varepsilon^{2 k-2}}(x, y, t, 0), \\
& d_{j 1}=\psi_{j, 1}(x, y, t, 0), \quad d_{j k}=\frac{\partial^{2 k-2} \psi_{j, 1}}{\partial \varepsilon^{2 k-2}}(x, y, t, 0), \\
& d_{j N+1}=\psi_{j, N+1}(x, y, t, 0), \quad d_{j N+k}=\frac{\partial^{2 k-2} \psi_{j, N+1}}{\partial \varepsilon^{2 k-2}}(x, y, t, 0), \\
& 2 \leq k \leq N, \quad 1 \leq j \leq 2 N,
\end{aligned}
$$

the functions $\varphi$ and $\psi$ being defined in (4.3).

Then the function defined by

$$
u(x, t)=-2\left(|v(x+3 t, y, t)|^{2}-1\right)
$$

is a solution to the KPI equation (1.1) depending on $2 N-2$ parameters $a_{k}, b_{k}, 1 \leq k \leq N-1$.

Proof. It is still a consequence of our previous works. Precisely, we have proven in [25] that the function $w$ defined by

$$
w(x, y)=\frac{\operatorname{det}\left(n_{j k}\right)_{j, k \in[1,2 N]_{t=0}}}{\operatorname{det}\left(d_{j k}\right)_{j, k \in[1,2 N]_{t}=0}} \times \exp (2 i y-i \varphi)
$$

is a solution to the nonlinear Schrödinger equation (4.8)

$$
i w_{y}+w_{x x}+2|w|^{2} w=0
$$

We can prove in the same way that the function $v$ defined by

$$
\tilde{v}(x, y, t)=v(x, y, t) \times \exp (2 i y-i \varphi)
$$

is a solution of the NLS equation by considering $t$ as a parameter. Then, we can deduce that the function $u$ defined by

$$
u(x, t)=-2\left(|v(x+3 t, y, t)|^{2}-1\right)=-2\left(|\tilde{v}(x+3 t, y, t)|^{2}-1\right)
$$

is a solution to the KPI equation which proves the result.

\section{The structure of the solutions to the KPI equation}

The structure of the rational solutions to the KPI equation is given by the following result

Theorem 5.1. Let $u$ the function defined by

$$
u(x, t)=-2\left(|v(x+3 t, y, t)|^{2}-1\right)=\frac{n(x, y, t)}{d(x, y, t)},
$$

with

$$
\begin{gathered}
v(x, y, t)=\frac{\operatorname{det}\left(\left(n_{j k}\right)_{j, k \in[1,2 N]}\right)}{\operatorname{det}\left(\left(d_{j k}\right)_{j, k \in[1,2 N]}\right)} \\
n_{j 1}=\varphi_{j, 1}(x, y, t, 0), \quad n_{j k}=\frac{\partial^{2 k-2} \varphi_{j, 1}}{\partial \varepsilon^{2 k-2}(x, y, t, 0),} \\
n_{j N+1}=\varphi_{j, N+1}(x, y, t, 0), \quad n_{j N+k}=\frac{\partial^{2 k-2} \varphi_{j, N+1}}{\partial \varepsilon^{2 k-2}}(x, y, t, 0), \\
d_{j 1}=\psi_{j, 1}(x, y, t, 0), \quad d_{j k}=\frac{\partial^{2 k-2} \psi_{j, 1}}{\partial \varepsilon^{2 k-2}(x, y, t, 0),} \\
d_{j N+1}=\psi_{j, N+1}(x, y, t, 0), \quad d_{j N+k}=\frac{\partial^{2 k-2} \psi_{j, N+1}}{\partial \varepsilon^{2 k-2}}(x, y, t, 0), \\
2 \leq k \leq N, \quad 1 \leq j \leq 2 N,
\end{gathered}
$$

Then the function $v$ is a rational solution to the KPI equation (1.1) quotient of two polynomials $n(x, y, t)$ and $d(x, y, t)$ depending on $2 N-2$ real parameters $a_{j}$ and $b_{j}, 1 \leq j \leq N-1$.

$n$ is a polynomial of degree $2 N(N+1)-2$ in $x, y$ and $t$.

$d$ is a polynomial of degree $2 N(N+1)$ in $x, y$ and $t$.

Proof. It is already proven in the previous section that this function is a solution to the KPI equation.

The proof of the structure of the solution is similar to this given in [26]. The difference in this present case is due to the reduction of the fraction which cancel the terms in $x^{2 N(N+1)}, y^{2 N(N+1)}, t^{2 N(N+1)}$ in the numerator, these terms having the same maximal power in numerator and denominator, and the fact that the elevation by the power 2 makes that the succeeding terms in $x, y$ and $t$ are to the power $2 N(N+1)-2$. 
Theorem 5.2. Let $u$ the function defined by

$$
u(x, t)=-2\left(|v(x+3 t, y, t)|^{2}-1\right)=\frac{n(x, y, t)}{d(x, y, t)},
$$

with

$$
\begin{gathered}
v(x, y, t)=\frac{\operatorname{det}\left(\left(n_{j k}\right)_{j, k \in[1,2 N]}\right)}{\operatorname{det}\left(\left(d_{j k}\right)_{j, k \in[1,2 N]}\right)} \\
n_{j 1}=\varphi_{j, 1}(x, y, t, 0), \quad n_{j k}=\frac{\partial^{2 k-2} \varphi_{j, 1}}{\partial \varepsilon^{2 k-2}(x, y, t, 0),} \\
n_{j N+1}=\varphi_{j, N+1}(x, y, t, 0), \quad n_{j N+k}=\frac{\partial^{2 k-2} \varphi_{j, N+1}}{\partial \varepsilon^{2 k-2}}(x, y, t, 0), \\
d_{j 1}=\psi_{j, 1}(x, y, t, 0), \quad d_{j k}=\frac{\partial^{2 k-2} \psi_{j, 1}}{\partial \varepsilon^{2 k-2}(x, y, t, 0),} \\
d_{j N+1}=\psi_{j, N+1}(x, y, t, 0), \quad d_{j N+k}=\frac{\partial^{2 k-2} \psi_{j, N+1}}{\partial \varepsilon^{2 k-2}}(x, y, t, 0), \\
2 \leq k \leq N, \quad 1 \leq j \leq 2 N,
\end{gathered}
$$

Then the function $v_{0}$ defined by

$$
v_{0}(x, y, t)=v(x, y, t)_{\left(a_{j}=b_{j}=0,1 \leq j \leq N-1\right)}
$$

is the solution of order $N$ solution to the KPI equation (1.1) whose highest amplitude in modulus is equal to $2(2 N+1)^{2}-2$.

Proof. The proof of this result is similar to this given in [26]. We do not give more details. The reader can do by himself the rewriting of this proof.

\section{Explicit expressions and patterns of the rational solutions to the KPI equation in function of parameters and time}

We have explicitly constructed rational solutions to the KPI equation of order $N$ depending on $2 N-2$ parameters for $1 \leq N \leq 3$.

In the following, we only give patterns of the modulus of the solutions in the plane $(x, y)$ of coordinates in function of the parameters $a_{i}$, and $b_{i}$, for $1 \leq i \leq N-1$ for $2 \leq N \leq 3$, and time $\mathrm{t}$.

We present the solutions using the following notations $X=2 x, Y=4 y, T=2 t$,

$$
u_{N}(X, Y, T)=1-\frac{G_{N}(X, Y, T)}{Q_{N}(X, Y, T)}
$$

with

$$
\begin{aligned}
& G_{N}(X, Y, T)=\sum_{k=0}^{2 N(N+1)} g_{k}(Y, T) X^{k}, \\
& Q_{N}(X, Y, T)=\sum_{k=0}^{2 N(N+1)} q_{k}(Y, T) X^{k} .
\end{aligned}
$$

By construction, all these solutions constructed in this study are real. Moreover, we know from the study of the NLS equation that the solutions constructed by ourself were non singular. From the construction, the denominators of the solutions to the KPI equation being the square of those of the solutions of the NLS equation, we get the non singularity of all these families of solutions to the KPI equation.

\subsection{Case $N=1$}

The polynomials $Q_{1}$ and $G_{1}$ are given by

$\mathbf{q}_{4}=1, \quad \mathbf{q}_{3}=-12 T, \quad \mathbf{q}_{2}=54 T^{2}+2 Y^{2}+2, \quad \mathbf{q}_{1}=-108 T^{3}+\left(-12 Y^{2}-12\right) T, \quad \mathbf{q}_{0}=81 T^{4}+Y^{4}+\left(18 Y^{2}+18\right) T^{2}+2 Y^{2}+1$

$\mathbf{g}_{4}=1, \quad \mathbf{g}_{3}=-12 T, \quad \mathbf{g}_{2}=54 T^{2}+2 Y^{2}-14, \quad \mathbf{g}_{1}=-108 T^{3}+\left(-12 Y^{2}+84\right) T, \quad \mathbf{g}_{0}=81 T^{4}+Y^{4}+\left(18 Y^{2}-126\right) T^{2}+18 Y^{2}+17$

This type of solution to the KPI equation is different from our previous works.

In our previous works [26-31], we constructed solution of order 1 to KPI equation and got

$\tilde{v}_{1}(X, Y, T)$

$=-2 \frac{9-6 X^{2}+72 X T+X^{4}+1296 T^{4}+216 X^{2} T^{2}-216 T^{2}+10 Y^{2}+Y^{4}-24 X T Y^{2}-24 X^{3} T+2 X^{2} Y^{2}-864 X T^{3}+72 T^{2} Y^{2}}{\left(X^{2}-12 X T+36 T^{2}+Y^{2}+1\right)^{2}}$.

The solution of order 1 obtained in this paper can be rewritten as

$v_{1}(X, Y, T)=16 \frac{-1+X^{2}-6 X T+9 T^{2}-Y^{2}}{\left.X^{2}-6 X T+9 T^{2}+Y^{2}+1\right)^{2}}=16 \frac{-1+(X-3 T)^{2}-Y^{2}}{\left(1+(X-3 T)^{2}+Y^{2}\right)^{2}}$.

It can be easily seen in this example that these two solutions are different and non singular. Moreover, we can verify that the maximum of the absolute value of $v_{1}$ is equal to $2(2 N+1)^{2}-2=16$ obtained when $X=Y=T=0$. 

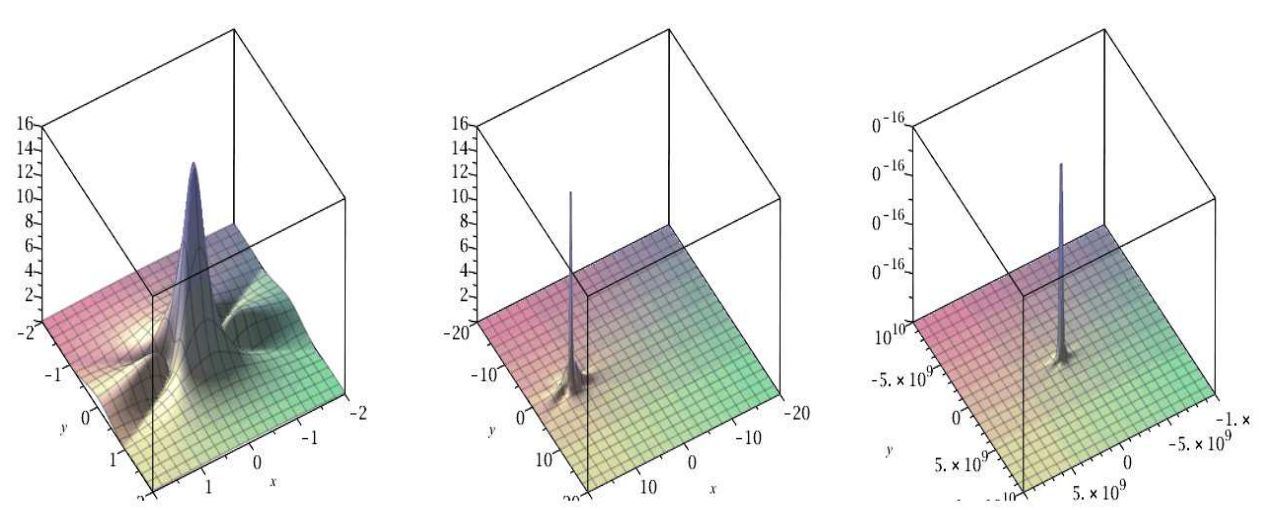

Figure 1. Solution of order 1 to the KPI equation, on the left for $t=0$; in the center for $t=4$; on the right for $t=10^{8}$.

\subsection{Case $N=2$}

In the case $N=2$, the polynomials $G_{2}$ and $G_{2}$ more complex are given by

$\mathbf{q}_{12}=, 1 \quad \mathbf{f}_{11}=-36 T, \quad \mathbf{q}_{10}=594 T^{2}+6 Y^{2}+6, \quad \mathbf{q}_{9}=-5940 T^{3}+\left(-180 Y^{2}+12\right) T-12 a_{1}, \quad \mathbf{q}_{8}=40095 T^{4}+6 Y^{4}+\left(2430 Y^{2}-\right.$ $2754) T^{2}+324 T a_{1}-36 Y^{2}+36 Y b_{1}+\left(3 Y^{2}+3\right)^{2}+54, \quad \mathbf{q}_{7}=-192456 T^{5}+\left(-19440 Y^{2}+42768\right) T^{3}-3888 T^{2} a_{1}+36 Y^{2} a_{1}+\left(-144 Y^{4}+\right.$ $\left.288 Y^{2}-864 Y b_{1}-1872+2\left(-36 Y^{2}+60\right)\left(3 Y^{2}+3\right)\right) T+36 a_{1}-12\left(3 Y^{2}+3\right) a_{1}, \quad \mathbf{q}_{6}=673596 T^{6}+2 Y^{6}+\left(102060 Y^{2}-333396\right) T^{4}+$ $27216 T^{3} a_{1}+54 Y^{4}-12 Y^{3} b_{1}+\left(1512 Y^{4}+3024 Y^{2}+9072 Y b_{1}+30312+2\left(162 Y^{2}-702\right)\left(3 Y^{2}+3\right)+\left(-36 Y^{2}+60\right)^{2}\right) T^{2}+198 Y^{2}-$ $108 Y b_{1}+54 a_{1}^{2}+18 b_{1}^{2}+\left(-756 Y^{2} a_{1}-1332 a_{1}-12\left(-36 Y^{2}+60\right) a_{1}+108\left(3 Y^{2}+3\right) a_{1}\right) T+2\left(3 Y^{4}-18 Y^{2}+18 Y b_{1}+27\right)\left(3 Y^{2}+\right.$ $3)+18, \quad \mathbf{q}_{5}=-1732104 T^{7}+\left(-367416 Y^{2}+1592136\right) T^{5}-122472 T^{4} a_{1}+\left(-9072 Y^{4}-54432 Y^{2}-54432 Y b_{1}-273456+2\left(162 Y^{2}-\right.\right.$ $\left.702)\left(-36 Y^{2}+60\right)+2\left(-324 Y^{2}+2268\right)\left(3 Y^{2}+3\right)\right) T^{3}+\left(6804 Y^{2} a_{1}+17172 a_{1}-324\left(3 Y^{2}+3\right) a_{1}-12\left(162 Y^{2}-702\right) a_{1}+108\left(-36 Y^{2}+\right.\right.$ $\left.60) a_{1}\right) T^{2}+\left(-36 Y^{6}-972 Y^{4}+216 Y^{3} b_{1}-3564 Y^{2}+1944 Y b_{1}-972 a_{1}^{2}-324 b_{1}^{2}+2\left(-18 Y^{4}-180 Y^{2}-108 Y b_{1}-450\right)\left(3 Y^{2}+3\right)-\right.$ $\left.324+2\left(3 Y^{4}-18 Y^{2}+18 Y b_{1}+27\right)\left(-36 Y^{2}+60\right)\right) T-12\left(3 Y^{4}-18 Y^{2}+18 Y b_{1}+27\right) a_{1}+2\left(18 Y^{2} a_{1}+18 a_{1}\right)\left(3 Y^{2}+3\right), \quad \mathbf{q}_{4}=3247695 T^{8}+$ $\left(918540 Y^{2}-4960116\right) T^{6}+367416 T^{5} a_{1}+\left(34020 Y^{4}+340200 Y^{2}+204120 Y b_{1}+1472580+\left(162 Y^{2}-702\right)^{2}+2\left(243 Y^{2}-2349\right)\left(3 Y^{2}+\right.\right.$ $\left.3)+2\left(-324 Y^{2}+2268\right)\left(-36 Y^{2}+60\right)\right) T^{4}+\left(-34020 Y^{2} a_{1}-12\left(-324 Y^{2}+2268\right) a_{1}-111780 a_{1}-324\left(-36 Y^{2}+60\right) a_{1}+108\left(162 Y^{2}-\right.\right.$ $\left.702) a_{1}+324\left(3 Y^{2}+3\right) a_{1}\right) T^{3}+\left(270 Y^{6}+7290 Y^{4}-1620 Y^{3} b_{1}+26730 Y^{2}-14580 Y b_{1}+7290 a_{1}^{2}+2430 b_{1}^{2}+2430+2\left(27 Y^{4}+702 Y^{2}+\right.\right.$ $\left.\left.162 Y b_{1}+3411\right)\left(3 Y^{2}+3\right)+2\left(-18 Y^{4}-180 Y^{2}-108 Y b_{1}-450\right)\left(-36 Y^{2}+60\right)+2\left(3 Y^{4}-18 Y^{2}+18 Y b_{1}+27\right)\left(162 Y^{2}-702\right)\right) T^{2}+$ $\left(-12\left(-18 Y^{4}-180 Y^{2}-108 Y b_{1}-450\right) a_{1}+108\left(3 Y^{4}-18 Y^{2}+18 Y b_{1}+27\right) a_{1}+2\left(-54 Y^{2} a_{1}-342 a_{1}\right)\left(3 Y^{2}+3\right)+2\left(18 Y^{2} a_{1}+18 a_{1}\right)\left(-36 Y^{2}+\right.\right.$ 60) $) T-12\left(18 Y^{2} a_{1}+18 a_{1}\right) a_{1}+2\left(Y^{6}+27 Y^{4}-6 Y^{3} b_{1}+99 Y^{2}-54 Y b_{1}+9 a_{1}^{2}+9 b_{1}^{2}+9\right)\left(3 Y^{2}+3\right)+\left(3 Y^{4}-18 Y^{2}+18 Y b_{1}+27\right)^{2}, \quad \mathbf{q} 3=$ $-4330260 T^{9}+\left(-1574640 Y^{2}+10182672\right) T^{7}-734832 T^{6} a_{1}+\left(-81648 Y^{4}-1143072 Y^{2}-489888 Y b_{1}-4856112+2\left(243 Y^{2}-2349\right)\left(-36 Y^{2}+\right.\right.$ $\left.60)+2\left(-324 Y^{2}+2268\right)\left(162 Y^{2}-702\right)\right) T^{5}+\left(102060 Y^{2} a_{1}+413100 a_{1}-324\left(162 Y^{2}-702\right) a_{1}+108\left(-324 Y^{2}+2268\right) a_{1}-12\left(243 Y^{2}-\right.\right.$ $\left.2349) a_{1}+324\left(-36 Y^{2}+60\right) a_{1}\right) T^{4}+\left(-1080 Y^{6}-29160 Y^{4}+6480 Y^{3} b_{1}-106920 Y^{2}+58320 Y b_{1}-29160 a_{1}^{2}-9720 b_{1}^{2}-9720+2\left(27 Y^{4}+\right.\right.$ $\left.702 Y^{2}+162 Y b_{1}+3411\right)\left(-36 Y^{2}+60\right)+2\left(-18 Y^{4}-180 Y^{2}-108 Y b_{1}-450\right)\left(162 Y^{2}-702\right)+2\left(-324 Y^{2}+2268\right)\left(3 Y^{4}-18 Y^{2}+18 Y b_{1}+\right.$ $27)) T^{3}+\left(-12\left(27 Y^{4}+702 Y^{2}+162 Y b_{1}+3411\right) a_{1}+108\left(-18 Y^{4}-180 Y^{2}-108 Y b_{1}-450\right) a_{1}-324\left(3 Y^{4}-18 Y^{2}+18 Y b_{1}+27\right) a_{1}+\right.$ $\left.2\left(-54 Y^{2} a_{1}-342 a_{1}\right)\left(-36 Y^{2}+60\right)+2\left(18 Y^{2} a_{1}+18 a_{1}\right)\left(162 Y^{2}-702\right)\right) T^{2}+\left(-12\left(-54 Y^{2} a_{1}-342 a_{1}\right) a_{1}+108\left(18 Y^{2} a_{1}+18 a_{1}\right) a_{1}+\right.$ $2\left(Y^{6}+27 Y^{4}-6 Y^{3} b_{1}+99 Y^{2}-54 Y b_{1}+9 a_{1}^{2}+9 b_{1}^{2}+9\right)\left(-36 Y^{2}+60\right)+2\left(-18 Y^{4}-180 Y^{2}-108 Y b_{1}-450\right)\left(3 Y^{4}-18 Y^{2}+18 Y b_{1}+\right.$ $27)) T-12\left(Y^{6}+27 Y^{4}-6 Y^{3} b_{1}+99 Y^{2}-54 Y b_{1}+9 a_{1}^{2}+9 b_{1}^{2}+9\right) a_{1}+2\left(18 Y^{2} a_{1}+18 a_{1}\right)\left(3 Y^{4}-18 Y^{2}+18 Y b_{1}+27\right), \quad \mathbf{q}_{2}=3897234 T^{10}+$ $\left(1771470 Y^{2}-13345074\right) T^{8}+944784 T^{7} a_{1}+\left(122472 Y^{4}+2204496 Y^{2}+734832 Y b_{1}+\left(-324 Y^{2}+2268\right)^{2}+9640296+2\left(243 Y^{2}-2349\right)\left(162 Y^{2}-\right.\right.$ $702)) T^{6}+\left(-183708 Y^{2} a_{1}-883548 a_{1}+324\left(162 Y^{2}-702\right) a_{1}+108\left(243 Y^{2}-2349\right) a_{1}-324\left(-324 Y^{2}+2268\right) a_{1}\right) T^{5}+\left(2430 Y^{6}+65610 Y^{4}-\right.$ $14580 Y^{3} b_{1}+240570 Y^{2}-131220 Y b_{1}+65610 a_{1}^{2}+21870 b_{1}^{2}+21870+2\left(27 Y^{4}+702 Y^{2}+162 Y b_{1}+3411\right)\left(162 Y^{2}-702\right)+2\left(243 Y^{2}-\right.$ $\left.2349)\left(3 Y^{4}-18 Y^{2}+18 Y b_{1}+27\right)+2\left(-18 Y^{4}-180 Y^{2}-108 Y b_{1}-450\right)\left(-324 Y^{2}+2268\right)\right) T^{4}+\left(324\left(3 Y^{4}-18 Y^{2}+18 Y b_{1}+27\right) a_{1}-\right.$ $324\left(-18 Y^{4}-180 Y^{2}-108 Y b_{1}-450\right) a_{1}+108\left(27 Y^{4}+702 Y^{2}+162 Y b_{1}+3411\right) a_{1}+2\left(-54 Y^{2} a_{1}-342 a_{1}\right)\left(162 Y^{2}-702\right)+2\left(18 Y^{2} a_{1}+\right.$ $\left.\left.18 a_{1}\right)\left(-324 Y^{2}+2268\right)\right) T^{3}+\left(-324\left(18 Y^{2} a_{1}+18 a_{1}\right) a_{1}+108\left(-54 Y^{2} a_{1}-342 a_{1}\right) a_{1}+\left(-18 Y^{4}-180 Y^{2}-108 Y b_{1}-450\right)^{2}+2\left(Y^{6}+\right.\right.$ $\left.\left.27 Y^{4}-6 Y^{3} b_{1}+99 Y^{2}-54 Y b_{1}+9 a_{1}^{2}+9 b_{1}^{2}+9\right)\left(162 Y^{2}-702\right)+2\left(27 Y^{4}+702 Y^{2}+162 Y b_{1}+3411\right)\left(3 Y^{4}-18 Y^{2}+18 Y b_{1}+27\right)\right) T^{2}+$ $\left(108\left(Y^{6}+27 Y^{4}-6 Y^{3} b_{1}+99 Y^{2}-54 Y b_{1}+9 a_{1}^{2}+9 b_{1}{ }^{2}+9\right) a_{1}+2\left(-54 Y^{2} a_{1}-342 a_{1}\right)\left(3 Y^{4}-18 Y^{2}+18 Y b_{1}+27\right)+2\left(18 Y^{2} a_{1}+\right.\right.$ $\left.\left.18 a_{1}\right)\left(-18 Y^{4}-180 Y^{2}-108 Y b_{1}-450\right)\right) T+2\left(Y^{6}+27 Y^{4}-6 Y^{3} b_{1}+99 Y^{2}-54 Y b_{1}+9 a_{1}^{2}+9 b_{1}^{2}+9\right)\left(3 Y^{4}-18 Y^{2}+18 Y b_{1}+27\right)+$ $\left(18 Y^{2} a_{1}+18 a_{1}\right)^{2}, \quad \mathbf{q}_{1}=-2125764 T^{11}+\left(-1180980 Y^{2}+10156428\right) T^{9}-708588 T^{8} a_{1}+\left(-104976 Y^{4}-2309472 Y^{2}-629856 Y b_{1}+\right.$ $\left.2\left(243 Y^{2}-2349\right)\left(-324 Y^{2}+2268\right)-10602576\right) T^{7}+\left(183708 Y^{2} a_{1}+324\left(-324 Y^{2}+2268\right) a_{1}-324\left(243 Y^{2}-2349\right) a_{1}+1023516 a_{1}\right) T^{6}+$ $\left(-2916 Y^{6}-78732 Y^{4}+17496 Y^{3} b_{1}-288684 Y^{2}+157464 Y b_{1}-78732 a_{1}^{2}-26244 b_{1}^{2}+2\left(243 Y^{2}-2349\right)\left(-18 Y^{4}-180 Y^{2}-108 Y b_{1}-\right.\right.$ $\left.450)+2\left(27 Y^{4}+702 Y^{2}+162 Y b_{1}+3411\right)\left(-324 Y^{2}+2268\right)-26244\right) T^{5}+\left(-324\left(27 Y^{4}+702 Y^{2}+162 Y b_{1}+3411\right) a_{1}+324\left(-18 Y^{4}-\right.\right.$ $\left.\left.180 Y^{2}-108 Y b_{1}-450\right) a_{1}+2\left(-54 Y^{2} a_{1}-342 a_{1}\right)\left(-324 Y^{2}+2268\right)+2\left(243 Y^{2}-2349\right)\left(18 Y^{2} a_{1}+18 a_{1}\right)\right) T^{4}+\left(-324\left(-54 Y^{2} a_{1}-342 a_{1}\right) a_{1}+\right.$ $324\left(18 Y^{2} a_{1}+18 a_{1}\right) a_{1}+2\left(Y^{6}+27 Y^{4}-6 Y^{3} b_{1}+99 Y^{2}-54 Y b_{1}+9 a_{1}^{2}+9 b_{1}{ }^{2}+9\right)\left(-324 Y^{2}+2268\right)+2\left(27 Y^{4}+702 Y^{2}+162 Y b_{1}+\right.$ $\left.3411)\left(-18 Y^{4}-180 Y^{2}-108 Y b_{1}-450\right)\right) T^{3}+\left(-324\left(Y^{6}+27 Y^{4}-6 Y^{3} b_{1}+99 Y^{2}-54 Y b_{1}+9 a_{1}^{2}+9 b_{1}{ }^{2}+9\right) a_{1}+2\left(-54 Y^{2} a_{1}-342 a_{1}\right)\left(-18 Y^{4}-\right.\right.$ $\left.\left.180 Y^{2}-108 Y b_{1}-450\right)+2\left(27 Y^{4}+702 Y^{2}+162 Y b_{1}+3411\right)\left(18 Y^{2} a_{1}+18 a_{1}\right)\right) T^{2}+\left(2\left(Y^{6}+27 Y^{4}-6 Y^{3} b_{1}+99 Y^{2}-54 Y b_{1}+9 a_{1}^{2}+\right.\right.$ $\left.\left.9 b_{1}^{2}+9\right)\left(-18 Y^{4}-180 Y^{2}-108 Y b_{1}-450\right)+2\left(-54 Y^{2} a_{1}-342 a_{1}\right)\left(18 Y^{2} a_{1}+18 a_{1}\right)\right) T+2\left(Y^{6}+27 Y^{4}-6 Y^{3} b_{1}+99 Y^{2}-54 Y b_{1}+\right.$ $\left.9 a_{1}^{2}+9 b_{1}^{2}+9\right)\left(18 Y^{2} a_{1}+18 a_{1}\right), \quad \mathbf{q}_{0}=531441 T^{12}+Y^{12}+\left(354294 Y^{2}-3424842\right) T^{10}+236196 T^{9} a_{1}+54 Y^{10}-12 Y^{9} b_{1}+\left(98415 Y^{4}-\right.$ $\left.118098 Y^{2}+236196 Y b_{1}+10491039\right) T^{8}-1259712 T^{7} a_{1}+927 Y^{8}-432 Y^{7} b_{1}+18 Y^{6} a_{1}^{2}+54 Y^{6} b_{1}{ }^{2}+\left(14580 Y^{6}+253692 Y^{4}+69984 Y^{3} b_{1}-\right.$ $\left.1495908 Y^{2}-839808 Y b_{1}+39366 a_{1}^{2}+13122 b_{1}^{2}-16011756\right) T^{6}+5364 Y^{6}-4104 Y^{5} b_{1}+486 Y^{4} a_{1}^{2}+1134 Y^{4} b_{1}^{2}-108 Y^{3} a_{1}^{2} b_{1}-$ $108 Y^{3} b_{1}{ }^{3}+\left(-17496 Y^{4} a_{1}+314928 Y^{2} a_{1}+52488 Y a_{1} b_{1}+2711880 a_{1}\right) T^{5}+\left(1215 Y^{8}+46332 Y^{6}+5832 Y^{5} b_{1}+598266 Y^{4}+229392 Y^{3} b_{1}-\right.$ $\left.13122 Y^{2} a_{1}^{2}+30618 Y^{2} b_{1}^{2}+4328316 Y^{2}+1358856 Y b_{1}-153090 a_{1}^{2}-42282 b_{1}^{2}+11592639\right) T^{4}+10287 Y^{4}-10800 Y^{3} b_{1}+1782 Y^{2} a_{1}^{2}+$ $4698 Y^{2} b_{1}{ }^{2}-972 Y a_{1}{ }^{2} b_{1}-972 Y b_{1}{ }^{3}+81 a_{1}{ }^{4}+162 a_{1}{ }^{2} b_{1}{ }^{2}+81 b_{1}{ }^{4}+\left(-2592 Y^{6} a_{1}-85536 Y^{4} a_{1}-19440 Y^{3} a_{1} b_{1}-816480 Y^{2} a_{1}-128304 Y a_{1} b_{1}+\right.$ 
$\left.2916 a_{1}{ }^{3}+2916 a_{1} b_{1}{ }^{2}-2330208 a_{1}\right) T^{3}+\left(54 Y^{10}+2862 Y^{8}+50076 Y^{6}-2592 Y^{5} b_{1}+3402 Y^{4} a_{1}{ }^{2}-1458 Y^{4} b_{1}{ }^{2}+323676 Y^{4}-84672 Y^{3} b_{1}+\right.$ $\left.49572 Y^{2} a_{1}{ }^{2}-4860 Y^{2} b_{1}{ }^{2}+2916 Y a_{1}{ }^{2} b_{1}+2916 Y b_{1}{ }^{3}+688014 Y^{2}-365472 Y b_{1}+178362 a_{1}{ }^{2}+61398 b_{1}{ }^{2}+61398\right) T^{2}+1782 Y^{2}-972 Y b_{1}+$ $162 a_{1}{ }^{2}+162 b_{1}{ }^{2}+\left(-108 Y^{8} a_{1}-3600 Y^{6} a_{1}+648 Y^{5} a_{1} b_{1}-29160 Y^{4} a_{1}+9936 Y^{3} a_{1} b_{1}-972 Y^{2} a_{1}{ }^{3}-972 Y^{2} a_{1} b_{1}{ }^{2}-68688 Y^{2} a_{1}+36936 Y a_{1} b_{1}-\right.$ $\left.6156 a_{1}^{3}-6156 a_{1} b_{1}^{2}-6156 a_{1}\right) T+81$

$\mathbf{g}_{12}=1, \quad \mathbf{g}_{12}=1, \quad \mathbf{g}_{11}=-36 T, \quad \mathbf{g}_{10}=594 T^{2}+6 Y^{2}-42, \quad \mathbf{g}_{9}=-5940 T^{3}+\left(-180 Y^{2}+1452\right) T-12 a_{1}, \quad \mathbf{g}_{8}=40095 T^{4}+15 Y^{4}+$ $\left(2430 Y^{2}-22194\right) T^{2}+324 T a_{1}-162 Y^{2}+36 Y b_{1}-81, \quad \mathbf{g}_{7}=-192456 T^{5}+\left(-19440 Y^{2}+198288\right) T^{3}-3888 T^{2} a_{1}+\left(-360 Y^{4}+3888 Y^{2}-\right.$ $\left.864 Y b_{1}+1944\right) T, \quad \mathbf{g}_{6}=673596 T^{6}+20 Y^{6}+\left(102060 Y^{2}-1149876\right) T^{4}+27216 T^{3} a_{1}-132 Y^{4}+96 Y^{3} b_{1}+\left(3780 Y^{4}-40824 Y^{2}+9072 Y b_{1}-\right.$ $6588) T^{2}-1728 T a_{1}-1476 Y^{2}+1152 Y b_{1}+54 a_{1}^{2}+18 b_{1}^{2}+1620, \quad \mathbf{g}_{5}=-1732104 T^{7}+\left(-367416 Y^{2}+4531464\right) T^{5}-122472 T^{4} a_{1}+$ $72 Y^{4} a_{1}+\left(-22680 Y^{4}+244944 Y^{2}-54432 Y b_{1}-126360\right) T^{3}+31104 T^{2} a_{1}+3888 Y^{2} a_{1}-216 Y a_{1} b_{1}+\left(-360 Y^{6}+1224 Y^{4}-1728 Y^{3} b_{1}-\right.$ $\left.35640 Y^{2}-17280 Y b_{1}-972 a_{1}^{2}-324 b_{1}^{2}-81000\right) T+3240 a_{1}, q u a d \mathbf{g}_{4}=3247695 T^{8}+15 Y^{8}+\left(918540 Y^{2}-12308436\right) T^{6}+367416 T^{5} a_{1}+$ $156 Y^{6}+72 Y^{5} b_{1}+\left(85050 Y^{4}-918540 Y^{2}+204120 Y b_{1}+1406970\right) T^{4}-233280 T^{3} a_{1}+8442 Y^{4}-3312 Y^{3} b_{1}-162 Y^{2} a_{1}^{2}+378 Y^{2} b_{1}{ }^{2}+$ $\left(2700 Y^{6}-540 Y^{4}+12960 Y^{3} b_{1}+692388 Y^{2}+103680 Y b_{1}+7290 a_{1}^{2}+2430 b_{1}^{2}+1286604\right) T^{2}+23004 Y^{2}-13176 Y b_{1}+1134 a_{1}^{2}+$ $2214 b_{1}^{2}+\left(-1080 Y^{4} a_{1}-53136 Y^{2} a_{1}+3240 Y a_{1} b_{1}-84888 a_{1}\right) T+1647, \quad \mathbf{g}_{3}=-4330260 T^{9}+\left(-1574640 Y^{2}+22779792\right) T^{7}-734832 T^{6} a_{1}+$ $96 Y^{6} a_{1}+\left(-204120 Y^{4}+2204496 Y^{2}-489888 Y b_{1}-6362712\right) T^{5}+933120 T^{4} a_{1}+1440 Y^{4} a_{1}+720 Y^{3} a_{1} b_{1}+\left(-10800 Y^{6}-32400 Y^{4}-\right.$ $\left.51840 Y^{3} b_{1}-4304016 Y^{2}-311040 Y b_{1}-29160 a_{1}^{2}-9720 b_{1}^{2}-8581680\right) T^{3}-864 Y^{2} a_{1}+4752 Y a_{1} b_{1}-108 a_{1}{ }^{3}-108 a_{1} b_{1}{ }^{2}+\left(6480 Y^{4} a_{1}+\right.$ $\left.287712 Y^{2} a_{1}-19440 Y a_{1} b_{1}+644112 a_{1}\right) T^{2}+\left(-180 Y^{8}-3408 Y^{6}-864 Y^{5} b_{1}-124344 Y^{4}+28224 Y^{3} b_{1}+1944 Y^{2} a_{1}^{2}-4536 Y^{2} b_{1}^{2}-\right.$ $\left.262224 Y^{2}+82080 Y b_{1}-8424 a_{1}^{2}-24840 b_{1}^{2}-144180\right) T+7776 a_{1}, \quad \mathbf{g}_{2}=3897234 T^{10}+6 Y^{10}+\left(1771470 Y^{2}-27516834\right) T^{8}+944784 T^{7} a_{1}+$ $270 Y^{8}+\left(306180 Y^{4}-3306744 Y^{2}+734832 Y b_{1}+15142788\right) T^{6}-2099520 T^{5} a_{1}+9468 Y^{6}-2592 Y^{5} b_{1}+378 Y^{4} a_{1}{ }^{2}-162 Y^{4} b_{1}{ }^{2}+\left(24300 Y^{6}+\right.$ $\left.150660 Y^{4}+116640 Y^{3} b_{1}+12763332 Y^{2}+466560 Y b_{1}+65610 a_{1}^{2}+21870 b_{1}^{2}+27660204\right) T^{4}+26460 Y^{4}-1728 Y^{3} b_{1}+2916 Y^{2} a_{1}^{2}-$ $4860 Y^{2} b_{1}{ }^{2}+324 Y a_{1}{ }^{2} b_{1}+324 Y b_{1}{ }^{3}+\left(-19440 Y^{4} a_{1}-769824 Y^{2} a_{1}+58320 Y a_{1} b_{1}-2087856 a_{1}\right) T^{3}+\left(810 Y^{8}+22248 Y^{6}+3888 Y^{5} b_{1}+\right.$ $\left.759996 Y^{4}-75168 Y^{3} b_{1}-8748 Y^{2} a_{1}^{2}+20412 Y^{2} b_{1}^{2}+1864296 Y^{2}+55728 Y b_{1}+14580 a_{1}^{2}+104004 b_{1}^{2}+2079594\right) T^{2}+51678 Y^{2}-$ $33696 Y b_{1}+3402 a_{1}^{2}+3078 b_{1}^{2}+\left(-864 Y^{6} a_{1}-25056 Y^{4} a_{1}-6480 Y^{3} a_{1} b_{1}-85536 Y^{2} a_{1}-53136 Y a_{1} b_{1}+972 a_{1}{ }^{3}+972 a_{1} b_{1}^{2}-178848 a_{1}\right) T-$ $8586, \quad \mathbf{g}_{1}=-2125764 T^{11}+\left(-1180980 Y^{2}+19604268\right) T^{9}-708588 T^{8} a_{1}+36 Y^{8} a_{1}+\left(-262440 Y^{4}+2834352 Y^{2}-629856 Y b_{1}-\right.$ $18738216) T^{7}+2519424 T^{6} a_{1}-144 Y^{6} a_{1}-216 Y^{5} a_{1} b_{1}+\left(-29160 Y^{6}-274104 Y^{4}-139968 Y^{3} b_{1}-18563256 Y^{2}-279936 Y b_{1}-78732 a_{1}{ }^{2}-\right.$ $\left.26244 b_{1}{ }^{2}-42766056\right) T^{5}+1080 Y^{4} a_{1}-5616 Y^{3} a_{1} b_{1}+324 Y^{2} a_{1}{ }^{3}+324 Y^{2} a_{1} b_{1}^{2}+\left(29160 Y^{4} a_{1}+1014768 Y^{2} a_{1}-87480 Y a_{1} b_{1}+2991816 a_{1}\right) T^{4}+$ $\left(-1620 Y^{8}-58320 Y^{6}-7776 Y^{5} b_{1}-2114424 Y^{4}+46656 Y^{3} b_{1}+17496 Y^{2} a_{1}^{2}-40824 Y^{2} b_{1}^{2}-7917264 Y^{2}-1127520 Y b_{1}+17496 a_{1}{ }^{2}-\right.$ $\left.192456 b_{1}{ }^{2}+527148\right) T^{3}-27216 Y^{2} a_{1}+8424 Y a_{1} b_{1}-2268 a_{1}{ }^{3}-2268 a_{1} b_{1}{ }^{2}+\left(2592 Y^{6} a_{1}+111456 Y^{4} a_{1}+19440 Y^{3} a_{1} b_{1}+785376 Y^{2} a_{1}+\right.$ $\left.190512 Y a_{1} b_{1}-2916 a_{1}{ }^{3}-2916 a_{1} b_{1}{ }^{2}-878688 a_{1}\right) T^{2}+\left(-36 Y^{10}-2196 Y^{8}-54504 Y^{6}+19008 Y^{5} b_{1}-2268 Y^{4} a_{1}{ }^{2}+972 Y^{4} b_{1}{ }^{2}-176040 Y^{4}+\right.$ $\left.100224 Y^{3} b_{1}-33048 Y^{2} a_{1}^{2}+23976 Y^{2} b_{1}^{2}-1944 Y a_{1}^{2} b_{1}-1944 Y b_{1}^{3}+125388 Y^{2}+67392 Y b_{1}+88452 a_{1}^{2}+17820 b_{1}^{2}+212220\right) T-$ $10044 a_{1}, \quad \mathbf{g}_{0}=, 531441 T^{12}+Y^{12}+\left(354294 Y^{2}-6259194\right) T^{10}+236196 T^{9} a_{1}+102 Y^{10}-12 Y^{9} b_{1}+\left(98415 Y^{4}-1062882 Y^{2}+236196 Y b_{1}+\right.$ $9546255) T^{8}-1259712 T^{7} a_{1}+1935 Y^{8}-432 Y^{7} b_{1}+18 Y^{6} a_{1}{ }^{2}+54 Y^{6} b_{1}{ }^{2}+\left(14580 Y^{6}+183708 Y^{4}+69984 Y^{3} b_{1}+10681308 Y^{2}+39366 a_{1}{ }^{2}+\right.$ $\left.13122 b_{1}{ }^{2}+25348788\right) T^{6}+2772 Y^{6}+2808 Y^{5} b_{1}-1674 Y^{4} a_{1}{ }^{2}-162 Y^{4} b_{1}{ }^{2}-108 Y^{3} a_{1}^{2} b_{1}-108 Y^{3} b_{1}{ }^{3}+\left(-17496 Y^{4} a_{1}-524880 Y^{2} a_{1}+\right.$ $\left.52488 Y a_{1} b_{1}-1487160 a_{1}\right) T^{5}+\left(1215 Y^{8}+54108 Y^{6}+5832 Y^{5} b_{1}+2176794 Y^{4}+42768 Y^{3} b_{1}-13122 Y^{2} a_{1}^{2}+30618 Y^{2} b_{1}{ }^{2}+12189852 Y^{2}+\right.$ $\left.1732104 Y b_{1}-48114 a_{1}^{2}+132678 b_{1}^{2}-11229921\right) T^{4}-6129 Y^{4}+30672 Y^{3} b_{1}-5994 Y^{2} a_{1}^{2}-13446 Y^{2} b_{1}^{2}+1620 Y a_{1}{ }^{2} b_{1}+1620 Y b_{1}{ }^{3}+$ $81 a_{1}^{4}+162 a_{1}{ }^{2} b_{1}{ }^{2}+81 b_{1}{ }^{4}+\left(-2592 Y^{6} a_{1}-147744 Y^{4} a_{1}-19440 Y^{3} a_{1} b_{1}-1562976 Y^{2} a_{1}-221616 Y a_{1} b_{1}+2916 a_{1}{ }^{3}+2916 a_{1} b_{1}{ }^{2}+\right.$ $\left.2708640 a_{1}\right) T^{3}+\left(54 Y^{10}+4158 Y^{8}+82908 Y^{6}-33696 Y^{5} b_{1}+3402 Y^{4} a_{1}^{2}-1458 Y^{4} b_{1}^{2}-138564 Y^{4}-312768 Y^{3} b_{1}+72900 Y^{2} a_{1}^{2}-\right.$ $\left.28188 Y^{2} b_{1}^{2}+2916 Y a_{1}{ }^{2} b_{1}+2916 Y b_{1}^{3}-2375730 Y^{2}+515808 Y b_{1}-171558 a_{1}^{2}-39690 b_{1}^{2}-186138\right) T^{2}+41958 Y^{2}-21708 Y b_{1}+$ $1458 a_{1}^{2}+4050 b_{1}^{2}+\left(-108 Y^{8} a_{1}-144 Y^{6} a_{1}+648 Y^{5} a_{1} b_{1}+50328 Y^{4} a_{1}+20304 Y^{3} a_{1} b_{1}-972 Y^{2} a_{1}^{3}-972 Y^{2} a_{1} b_{1}{ }^{2}+273456 Y^{2} a_{1}-\right.$ $\left.77112 Y a_{1} b_{1}+1620 a_{1}^{3}+1620 a_{1} b_{1}^{2}-16524 a_{1}\right) T+3969$

For $N=2$, the formation of three peaks is obtained when the parameters $a_{1}$ or $b_{1}$ are not equal to 0 .
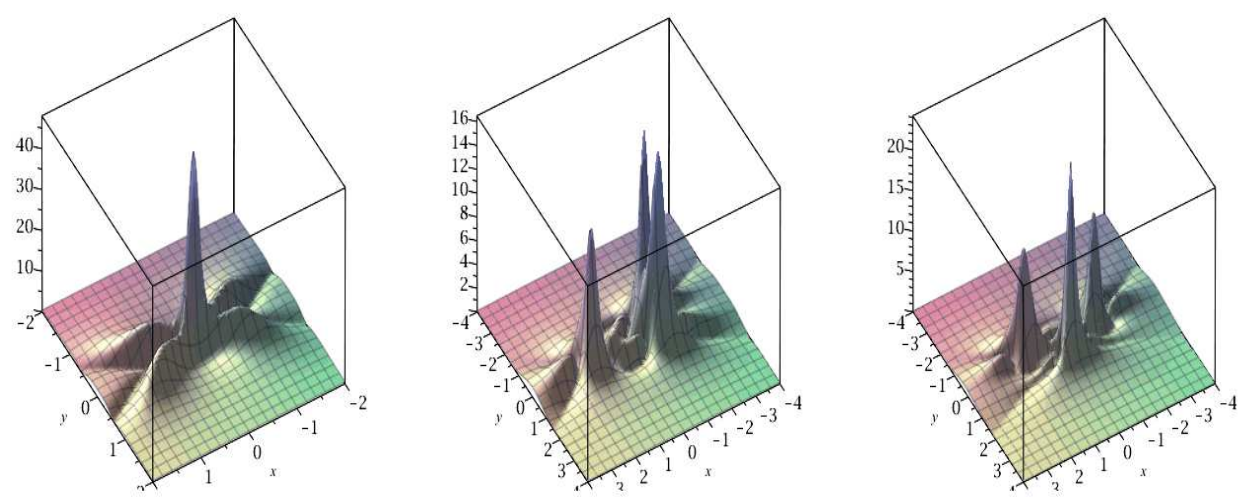

Figure 2. Solution of order 2 to the KPI equation for $t=0$, on the left $a_{1}=0, b_{1}=0$,; in the center $a_{1}=10, b_{1}=0$,; on the right $a_{1}=10, b_{1}=10$. 

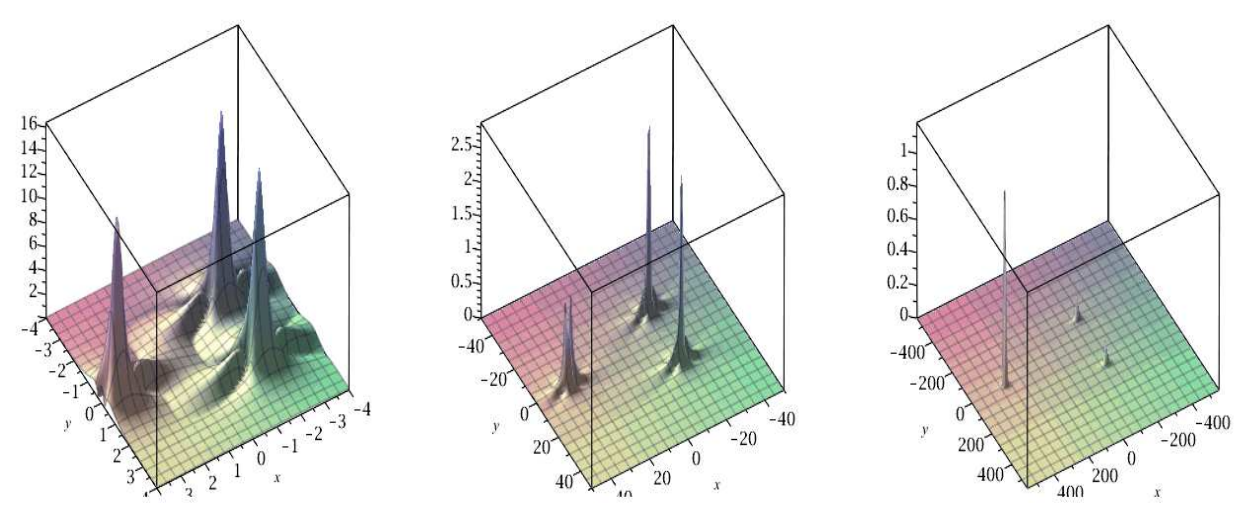

Figure 3. Solution of order 2 to the KPI equation for $t=0$, on the left $a_{1}=10^{2}, b_{1}=0$; in the center $a_{1}=10^{4}, b_{1}=0$; on the right for $t=10, a_{1}=10^{8}, b_{1}=0$.
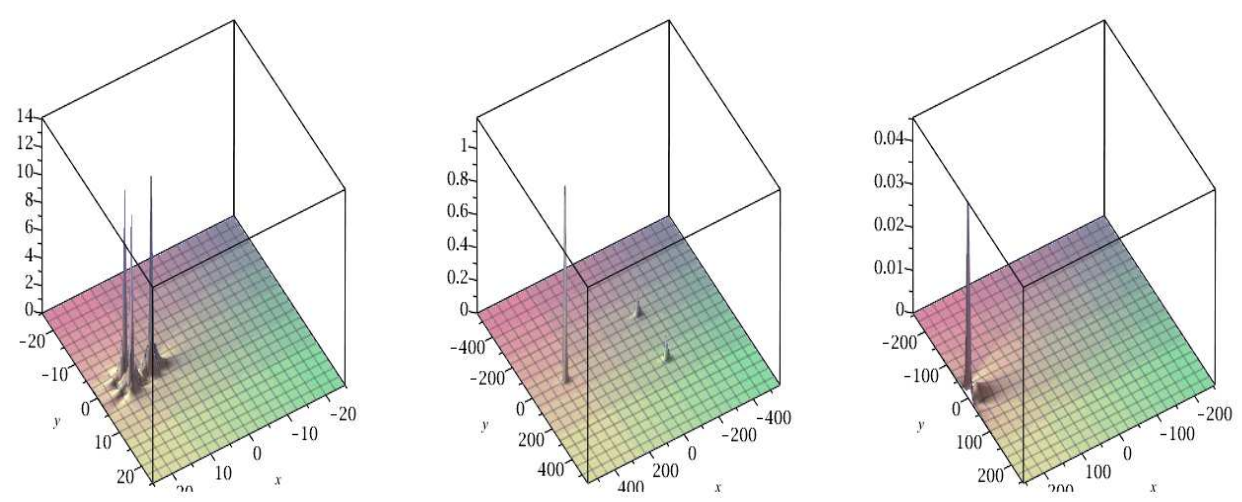

Figure 4. Solution of order 2 to the KPI equation, on the left for $t=5, a_{1}=0, b_{1}=0$; in the center for $t=10, a_{1}=0, b_{1}=0$; on the right for $t=100, a_{1}=0, b_{1}=0$.

\subsection{Case $N=3$}

In this case, polynomials $G_{3}$ and $Q_{3}$ depending on 4 parameters being too complex, we cannot give their explicit expressions. Even without parameters, due to the length of the solution, the explicit expression cannot be given here.

In the case $N=3$, for $a_{1} \neq 0$ or $b_{1} \neq 0$ and the other parameters equal to zero, we obtain a triangle with 6 peaks; for $a_{2} \neq 0$ or $b_{2} \neq 0$, and other parameters equal to zero, we obtain a concentric rings of 5 peaks with a peak in the center.
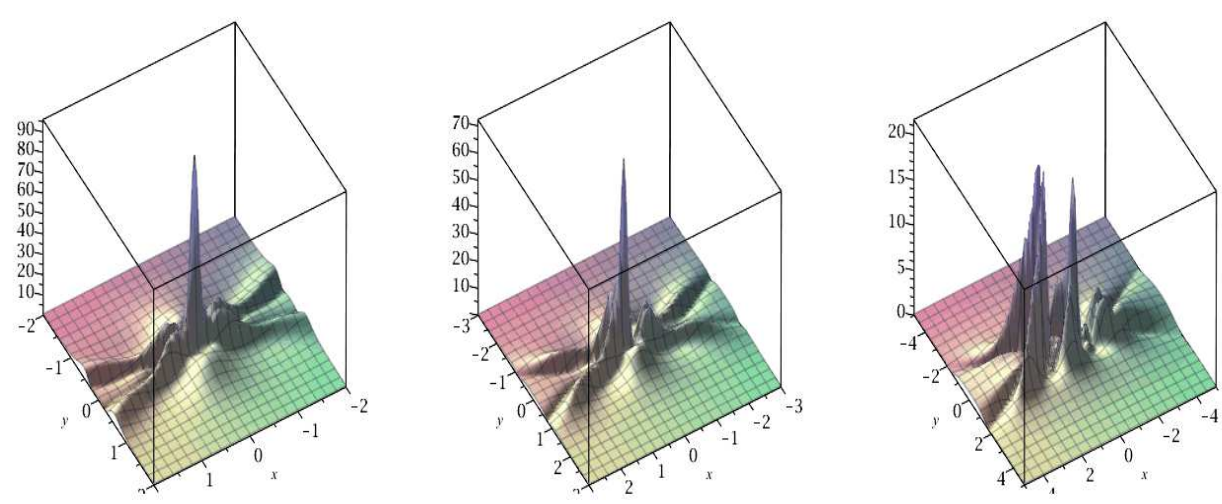

Figure 5. Solution of order 3 to the KPI equation, on the left for $t=0$; in the center for $t=0,01$; on the right for $t=0,1$; all the parameters are equal to 0 . 

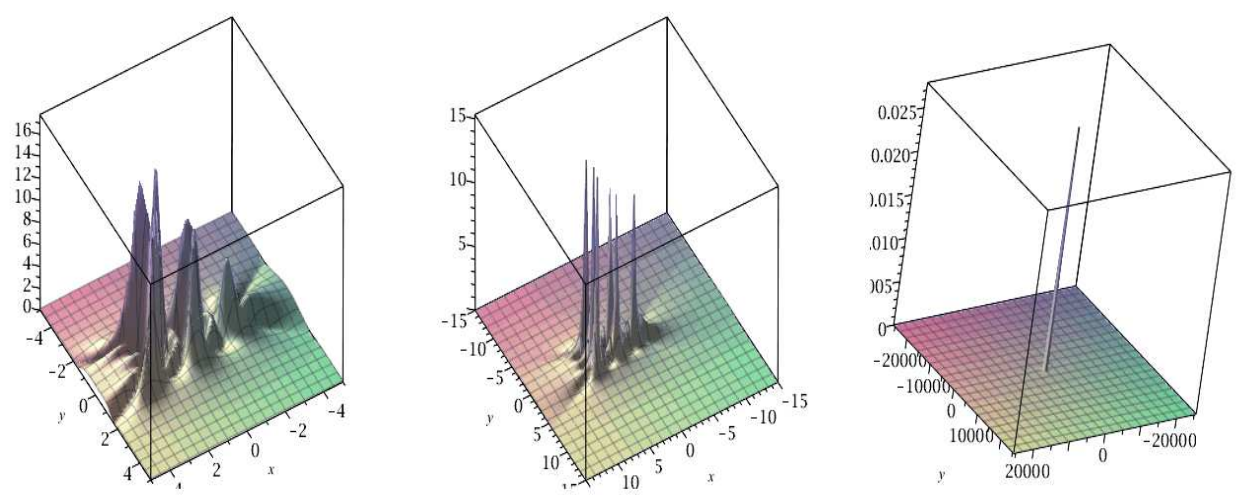

Figure 6. Solution of order 3 to the KPI equation, on the left for $t=0,2$; in the center for $t=10^{0}$; on the right for $t=10^{1}$; all the parameters are equal to 0 .
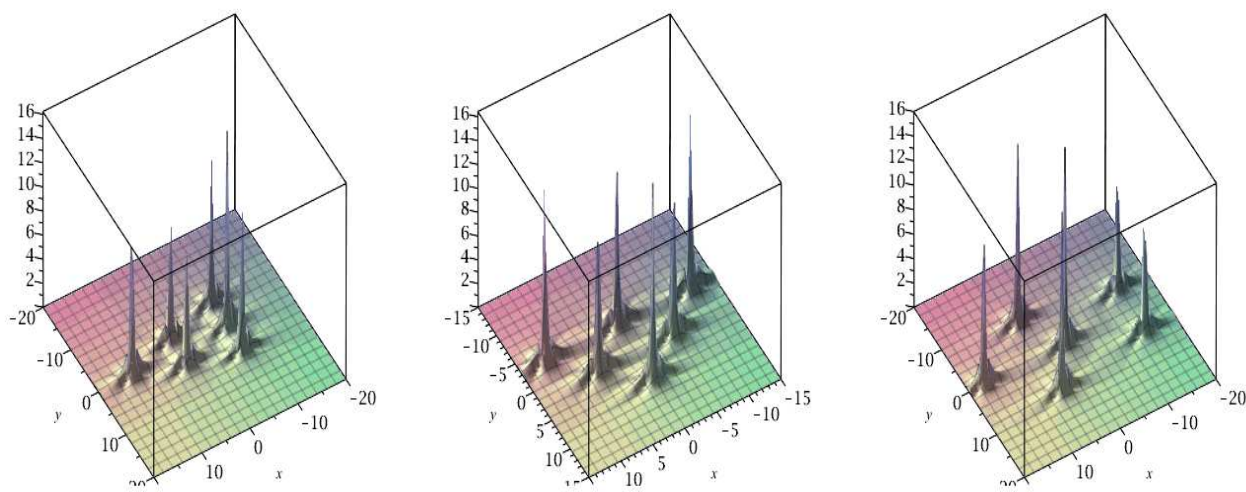

Figure 7. Solution of order 3 to the KPI equation, on the left for $a_{1}=10^{3}$; in the center for $b_{1}=10^{3}$; on the right for $a_{2}=10^{6}$; here $t=0$.
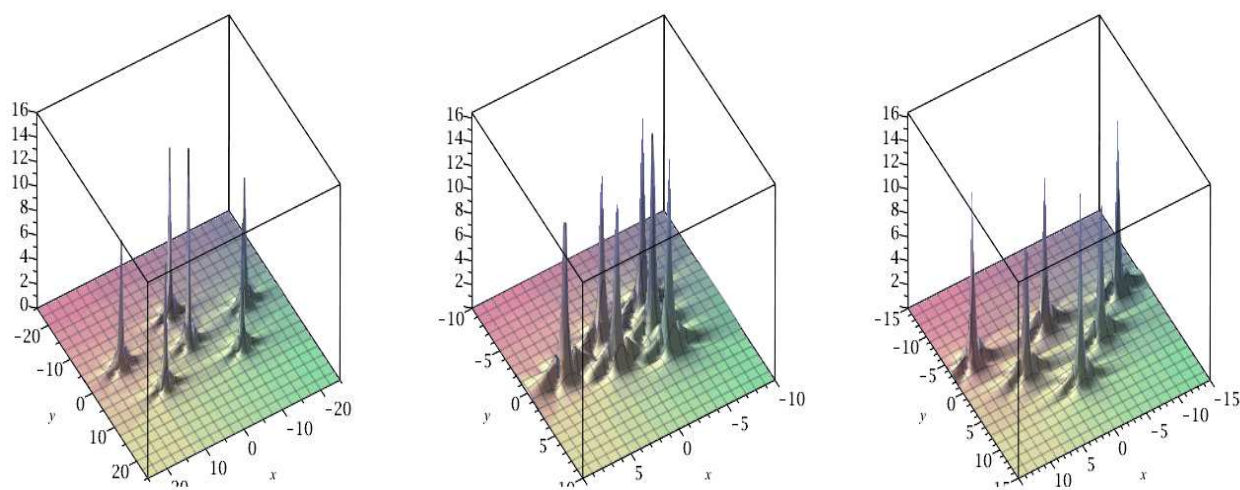

Figure 8. Solution of order 3 to the KPI equation, on the left for $t=0, b_{2}=10^{6}$; in the center for $t=0,01, a_{1}=10^{3}$ all the other parameters are equal to 0 ; on the right for $t=0,1, b_{1}=10^{3}$ all the parameters are equal to 0 .

\section{Conclusion}

We have given three representations of the solutions to the KPI equation: in terms of Fredholm determinants of order $2 N$ depending on $2 N-1$ real parameters in function of exponentials; in terms of wronskians of order $2 N$ depending on $2 N-1$ real parameters by means of trigonometric functions; in terms of real rational solutions as a quotient of two polynomials $n(x, y, t)$ and $d(x, y, t)$ of degrees $2 N(N+1)-2$ in $x, y, t$ and $2 N(N+1)$ in $x, y, t$ respectively and depending on $2 N-2$ real parameters $a_{j}$ and $b_{j}, 1 \leq j \leq N-1$.

The maximum of the modulus of those solutions is equal to $2(2 N+1)^{2}-2$. That gives a new approach to find explicit solutions for higher orders and try to describe the structure of those rational solutions.

In the $(x, y)$ plane of coordinates, different structures appear.

All the solutions described in this study are different from those constructed in previous works [26-31] .

It will be relevant to go on this study for higher orders to try to understand the structure of those rational solutions.

\section{Acknowledgements}

The author would like to thank the referees for their suggestions and comments which improve the original version of the paper. 


\section{Funding}

There is no funding for this work.

\section{Availability of data and materials}

Not applicable.

\section{Competing interests}

The authors declare that they have no competing interests.

\section{Author's contributions}

All authors contributed equally to the writing of this paper. All authors read and approved the final manuscript.

\section{References}

[1] B.B. Kadomtsev, V.I. Petviashvili, On the stability of solitary waves in weakly dispersing media, Sov. Phys. Dokl., V. 15, N. 6, (1970), 539-541

[2] M.J. Ablowitz, H. Segur On the evolution of packets of water waves, J. Fluid Mech., V. 92, (1979), 691-715

[3] D.E. Pelinovsky, Y.A. Stepanyants, Y.A. Kivshar, Self-focusing of plane dark solitons in nonlinear defocusing media, Phys. Rev. E, V. 51, (1995), 5016-5026

[4] V.S. Dryuma, On analytical solutions of the two-dimensional Korteweg-de Vries equation, Pisma Zh. Teor. Fiz., V19, N. 12, (1973), 219-225

[5] S.V. Manakov, V.E. Zakharov, L.A. Bordag, V.B. Matveev, Two-dimensional solitons of the Kadomtsev-Petviashvili equation and their interaction, Phys. Letters, V. 63A, N. 3, (1977), 205-206

[6] I. Krichever, Rational solutions of the Kadomtcev-Petviashvili equation and integrable systems of $n$ particules on a line, Funct. Anal. and Appl., V. 12 , N. 1, (1978), 76-78

[7] I. Krichever, S. Novikov Holomorphic bundles over riemann surfaces and the kadomtsev-petviashvili equation, Funkt. Ana. E Pril., V. 12, N.4, (1978),

[8] B.A. Dubrovin Theta functions and non-linear equations, Russian Math. Surveys, V.36, N.2, (1981), 11-92

[9] I. Krichever, Elliptic solutions of the KP equation and integrable systems of particles, Funkt. Ana. E Pril., V. 14, N.4, (1990), 45-54

[10] J. Satsuma, M.J. Ablowitz, Two-dimensional lumps in nonlinear dispersive systems, J. Math. Phys., V. 20, (1979), 1496-1503

[11] V.B. Matveev, Darboux transformation and explicit solutions of the Kadomtcev-Petviaschvily equation depending on functional parameters, Letters in Mathematical Physics, V. 3, (1979), 213-216

[12] N. C Freeman, J.J.C. Nimmo, Rational solutions of the KdV equation in wronskian form, Phys. Letters, V. 96 A, N. 9, (1993), $443-446$

[13] N. C Freeman, J.J.C. Nimmo The use of Bäcklund transformations in obtaining N-soliton solutions in wronskian form, J. Phys. A : Math. Gen., V. 17 A, (1994), 1415-1424

[14] V.B. Matveev, M.A. Salle, New families of the explicit solutions of the Kadomtcev-Petviaschvily equation and their application to Johnson equation, Proceedings, Some Topics On Inverse Problems, World Scientific, (1987), 304-315

[15] D.E. Pelinovsky, Y.A. Stepanyants, New multisolitons of the Kadomtsev-Petviashvili equation, Phys. JETP Lett., V. 57, (1993), 24-28

[16] D.E. Pelinovsky, Rational solutions of the Kadomtsev-Petviashvili hierarchy and the dynamics of their poles. I. New form of a general rational solution, J.Math.Phys., V. 35, (1994), 5820-5830

[17] M.J Ablowitz, J. Villarroel, Solutions to the time dependent schrödinger and the Kadomtsev-Petviashvili equations, Phys. Rev. Lett., V. 78, (1997), 570

[18] J. Villarroel, M.J Ablowitz, On the discrete spectrum of the nonstationary Schrdinger equation and multipole lumps of the Kadomtsev-Petviashvili 1 equation, Commun. Math. Phys., V. 207, (1009), 1-42

[19] M.J. Ablowitz, S. Chakravarty, A.D. Trubatch, J. Villaroel, A novel class of solution of the non-stationary Schrödinger and the KP equations, Phys. Let. A, V. 267, (2000), 132-146

[20] G. Biondini, Y. Kodama, On a family of the Kadomtsev-Petvisvili equation which also satisfy the Toda lattice hierarchy, J. Phys. A: Math. Gen., V. 36, (2003), 10519-10536

[21] Y. Kodama, Young diagrams and N solitons solutions to the KP equation, J. Phys. A: Math. Gen., V. 37, (2004), 11169-11190

[22] G. Biondini, Line Soliton Interactions of the Kadomtsev-Petviashvili Equation, PRL, V. 99, (2007), 064103-1-4

[23] P. Gaillard, Families of quasi-rational solutions of the NLS equation and multi-rogue waves, J. Phys. A : Meth. Theor., V. 44, (2011), 1-15

[24] P. Gaillard, Wronskian representation of solutions of the NLS equation and higher Peregrine breathers, J. Math. Sciences : Adv. Appl., V. 13, N. 2, (2012), 71-153

[25] P. Gaillard, Degenerate determinant representation of solution of the NLS equation, higher Peregrine breathers and multi-rogue waves, J. Math. Phys., V. 54, (2013), 013504-1-32

[26] P. Gaillard, Multiparametric families of solutions of the KPI equation, the structure of their rational representations and multi-rogue waves., Theo. And Mat. Phys., V. 196, N. 2, (2018), 1174-1199

[27] P. Gaillard, Rational solutions to the KPI equation and multi rogue waves, Annals Of Physics, V. 367, (2016), 1-5

[28] P. Gaillard, Fredholm and Wronskian representations of solutions to the KPI equation and multi-rogue waves, Jour. of Math. Phys., V. 57, (2016), $063505-1-13$

[29] P. Gaillard, From Fredholm and Wronskian representations to rational solutions to the KPI equation depending on $2 N-2$ parameters, Int. Jour. of Appl. Sci. And Math., V. 4, N. 3, (2017), 60-70

[30] P. Gaillard, Families of Rational Solutions of Order 5 to the KPI Equation Depending on 8 Parameters, New Hor. in Math. Phys., V. 1, N. 1, (2017), 26-31

[31] P. Gaillard, 6-th order rational solutions to the KPI Equation depending on 10 parameters, Jour. Of Bas. And Appl. Res. Int., V. 21, N. 2, (2017), 92-98 